\title{
Erratum to: Mathematical working space and paradigms as an analysis tool for the teaching and learning of analysis
}

\author{
Elizabeth Montoya Delgadillo ${ }^{1} \cdot$ Laurent $_{\text {Vivier }}{ }^{2,3}$
}

Published online: 1 July 2016

(C) FIZ Karlsruhe 2016

\section{Erratum to: ZDM Mathematics Education DOI 10.1007/s11858-016-0777-9}

The authors very much regret that errors have slipped into their contribution to the special issue on "Mathematical working spaces in schooling". The original article was corrected.

The corrections are listed below:

\subsection{Two global paradigms}

In the 4th paragraph the first sentence should read:

It is moreover significant that approximately $60 \%$ of mathematics subjects (both students and teachers) consistently answer that the comparison of $0.999 \ldots$ and 1 is an inequality, independently of period, country or any preliminary teaching (Tall 1980; Mena-Lorca, Mena-Lorca, Montoya-Delgadillo, Morales and Parraguez 2015; Rittaud and Vivier 2014).

The online version of the original article can be found under doi:10.1007/s11858-016-0777-9.

Laurent Vivier

laurent.vivier@univ-paris-diderot.fr

Elizabeth Montoya Delgadillo

elizabeth.montoya@pucv.cl

1 IMA, Pontificia Universidad Católica de Valparaíso, Valparaíso, Chile

2 LDAR, Université Paris Diderot, Paris, France

3 IMAG, Université de Montpellier, Montpellier, France

\subsection{Description of work in the various paradigms}

The beginning should read:

In the GA paradigm, visualization alone is sufficient, possibly supplemented by zooms enabled by the artifact (and perhaps by knowledge of micro-straightness according to Maschietto (2002), where a regular curve and its tangent are locally indiscernible). Visualization can also be used to establish a conjecture-justified in the CA or RA paradigm with a dialectic between paradigms-, as shown by Kuzniak (2004) for GI and GII.

In the 9th paragraph the 1st sentence should read:

Likewise, we obtain $d t / d y=0$ at $y=0$, but the composition of derivatives (such as $d m / d t=d m / d y \times d y / d t$ ) leads to a division by zero.

In the last paragraph the 2nd sentence should read:

For example, we may write, without reflection, $d t /$ $d m=d y / d m \times d t / d y=4 /(3 a) \times 0=0$.

\section{Conclusion}

The 3rd paragraph should read:

We pointed out the specific structure of paradigms of analysis with Standard Analysis and Non Standard Analysis: teaching institutions refer mainly to the former. Three paradigms were then proposed and we showed, in examples, how they are useful and how they orientate the work in analysis. 